\title{
Modeling Seepage Behavior of Smaller Vehicles in Mixed Traffic Conditions Using an Agent Based Simulation
}

\author{
Amit Agarwal $^{1} \cdot$ Gregor Lämmel $^{2,3}$
}

Received: 17 September 2015/Accepted: 4 April 2016/Published online: 19 April 2016

(C) Springer International Publishing Switzerland 2016

\begin{abstract}
Seepage is an important yet rarely modeled or quantified phenomenon in mixed traffic streams. It describes situations where smaller vehicles do not line up but rather "seep" through a queue of stationary or almost stationary vehicles. This contribution introduces seepage into an agent-based transport simulation model. In order to allow vehicles to seep, the traditional first-in-first-out queue model is modified such that in the free flow regime, faster vehicles can overtake slower vehicles and in the congested regime, slower vehicles can overtake faster vehicles. The model is validated with the help of fundamental diagrams. Its sensitivity is investigated by comparing the impact of different shares of smaller vehicles on the speed-density relation in the mixed traffic streams. A case study of the evacuation of Patna, India under mixed traffic conditions with seepage demonstrates the overall approach.
\end{abstract}

Keywords Seepage $\cdot$ Lane splitting $\cdot$ Lane sharing - Lane filtering · Mixed traffic · Agent-based modeling · Backward traveling holes $\cdot$ Evacuation

Amit Agarwal

amit.agarwal@campus.tu-berlin.de

1 Technische Universität Berlin, Sekr. SG 12, Salzufer 17-19, 10587 Berlin, Germany

2 Forschungszentrum Jülich, Institute for Advance Simulation, Jülich, Germany

3 German Aerospace Center (DLR), Institute of Transport Systems, Rutherfordstr. 2, 12489 Berlin, Germany

\section{Introduction}

Modeling complex heterogeneous traffic is a challenge for traffic planners since streets are full of variety of vehicles. These vehicles can be differentiated based on their static (physical dimension) and dynamic (speed, acceleration, power etc.) characteristics. The traffic in industrializing nations is different to the traffic in industrialized nations for at least following reasons (a) absence of lane discipline (b) common road space for motorized and non-motorized vehicles. The motorized vehicles include car, bus, truck, motorbike (motorcycles), scooters and, three-wheeler motorized rickshaws and non-motorized vehicles include bikes (bicycles), cycle-rickshaws etc. The presence of mixed traffic imply a different driving behavior than it is observed under homogeneous conditions. This contribution investigates one of such, so-called seepage behavior, which predominantly exists in most of the industrializing nations.

Due to its size and better maneuverability, smaller vehicles (motorbike, bike etc.) are less sensitive to the remaining traffic but in turn, affect flow of other vehicles considerably. Thus, during queue built up or at traffic signals, these vehicles move continuously across the gaps between stationary vehicles and come in front of the queues. In literature, this behavior is known as 'seepage action' $[1,2]$, lane filtering (passing between stationary vehicles), lane splitting (passing between moving traffic) [3], and lane sharing [4].

Figure 1a shows seepage behavior of motorbikes and bikes at a traffic light in India, Fig. 1b illustrates the differences between passing and seepage behavior, and Fig. 1c exemplifies seepage of a bike and a motorbike at a traffic light. The bike (green dashed line) does not line up behind the cars when approaching the red traffic light, instead it seeps across the available gaps to come in front of 


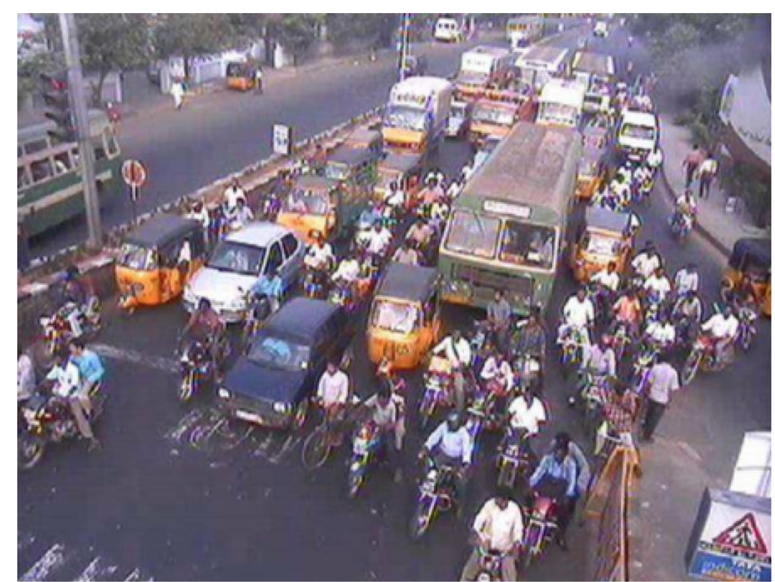

(a) Seepage of smaller vehicles at traffic signal [6].

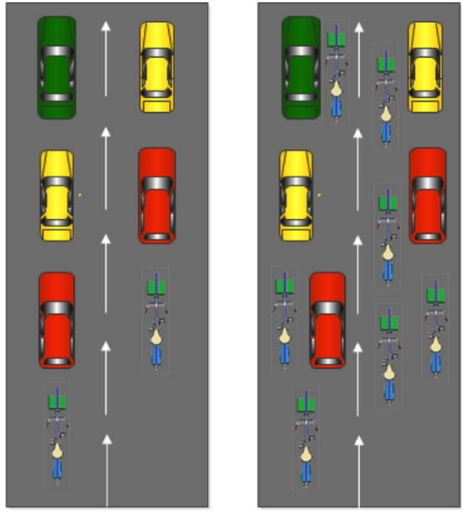

(b) Passing and seepage.

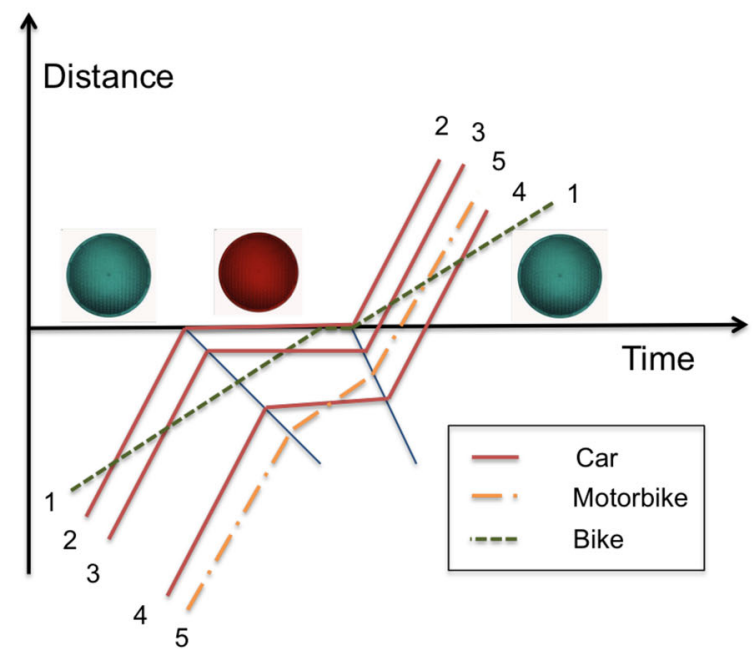

(c) Schematic of seepage at traffic signal. Reproduced after Oketch(2000) [1].

Fig. 1 Seepage action

the queue. Consequently, it departs before the cars when the traffic light turns green [1,5]. Similarly, the motorbike (in orange dash-dot line) also seeps through the queue of cars. This behavior is a common praxis in the developing nations.

Studies that focus on the modeling of such traffic behavior are sparse. There exists, however, a large body of works that investigate pros and cons of it. Aupetit et al. [7] find that lane splitting is a systematic practice in the Paris region.

On the practical implementation side, from July 2014, lane filtering is legally approved in New South Wales, Australia under the Road Transport Legislation Amendment (Lane Use by Motor Bikes) Regulation 2014 [8]. By allowing lane filtering, the authorities aim for less congestion and fewer rear end collisions. A detailed overview on the related studies is given in Agarwal and Lämmel [9]. The present work focuses on the modeling of seepage in the context of a microscopic agent-based simulation.
Oketch [1] makes an attempt to implement this behavior using lateral movement model. Nair et al. [10] present an analogues multi-class 'porous model' to allow for seepage, in which traffic stream is considered as a porous medium and each vehicle type represents a class. Each vehicle class is considered to move through a series of pores and speed is determined by the availability of pores. In another study, Asaithambi et al. [6] address the issue of seepage of motorbikes at traffic signals. The authors use exclusive stopping space for motorcycles (ESSM) in front of the queue at intersections and find it beneficial for all modal split except when the share of cars is dominant in traffic composition. Similarly, Fan and Work [11, 12] develop a seepage model as a multi-class generalization of the cell transmission model. The models in the above studies are highly detailed models. Consequently, they are CPU-intensive and unsuitable for simulating large-scale scenarios. A previous work [9] introduces seepage functionality in a multi agent travel demand 
simulator MATSim [13]. The seepage behavior is validated with the help of fundamental diagrams (FDs) and spatiotemporal plots. But the congested branch of the FDs for seepage remains unclear in [9]. Therefore, in the present study, the authors wish to extend and validate the seepage behavior with the help of a real-world scenario in addition to the fundamental diagrams.

A related situation is the evacuation of large urban areas, e.g. in the case of tsunamis. ${ }^{1}$ As evacuees usually want to exit the affected area as fast as possible it is expected that seepage situations occur. This situation is addressed in a small evacuation experiment, where pedestrians are evacuated from an open ground to an exit zone (safe place) connected by a narrow street. The seepage behavior of pedestrians is studied under different mixing ratio of stationary cars [15]. The existing simulation framework (MATSim) is able to model the large evacuation problems $[16,17]$ in homogeneous traffic conditions. To the authors knowledge there exist no other simulation framework to model the seepage behavior under the highly dynamic conditions observed in the real-world traffic in general and in particular in case of evacuations. With the help of the proposed approach, it will be possible to simulate large-scale evacuation scenarios under mixed traffic conditions and allowing seepage behavior. It is assumed that evacuation model also would be more realistic from seepage. The results are quantified with the help of a realworld scenario of Patna, India.

The present study aims to: (1) extend the seepage queue model for multiple seep modes, (2) validate it with the help of the fundamental diagrams mainly in congested regime and test the sensitivity, (3) show the suitability for largescale scenario simulation and quantify the benefits.

The remainder of the paper is organized as follows. The next section briefs about the travel demand simulator and seepage queue model followed by which validation of the proposed model is done with the help of fundamental diagrams from different traffic mixes. The subsequent section presents sensitivity analysis of the model and a real-word scenario is presented after it. Before the concluding section, some additional aspects are discussed.The study is concluded in the final section.

\section{Modeling}

\section{Travel Demand Simulator: MATSim}

The multi-agent transport simulation framework, MATSim [13] is used for all simulation experiments in the

\footnotetext{
${ }^{1}$ See, e.g. [14] for a detailed overview of problems that arise when planing the evacuation of whole cities.
}

present study. Detailed information about the software has been published in several studies see e.g. [18-21].

Inputs A simulation run minimally requires three inputs. (1) Physical boundary conditions (i.e. road network), (2) daily plans of individual travelers and (3) configuration parameters. These inputs are converted into the requisite format.

Simulation framework The simulation framework is composed of following three steps:

1. Plans execution Daily plans of all agents are loaded simultaneously using a network loading algorithm. This is embedded into an iterative co-evolutionary algorithm [18]. The network loading algorithm of the MATSim framework is a computationally efficient queue model [22-24]. By default, the state-of-the-art first-in-first-out queue model is used. However, similar to previous studies [9, 25, 26], in the present study also, the queue model is further modified for various functionalities.

2. Plans evaluation In order to compare between the plans of an agent, executed plans are evaluated using a utility function. In this study, MATSim standard 'Charypar-Nagel' scoring function is used [27].

3. Re-planning In the simulation framework, every agent learns and adapts to the system. Therefore, a new plan is generated for some of the agents by modifying an existing plan's attribute (departure time, route, mode etc.) using so called innovative strategies. The new plan then is executed in the next iteration.

By repeatedly performing the steps above, an iterative process is initiated which results in the stabilized simulation outputs.

\section{Seepage methodology}

Historically, MATSim simulates the traffic flow of vehicles by a first in first out (FIFO) queue model [22, 23]. Agarwal et al. [25, 28] propose an earliest-link-exit-time approach that softens the strict FIFO order of the simulated vehicles. The earliest link exit time $\left(t_{\text {earliest }}\right)$ on a link is given by Eq. 1, where $\ell_{l}$ is length of the link $l, v_{l, \max }$ is maximum speed allowed on the link, and $v_{v, \max }$ is maximum speed of the vehicle. The vehicles on the link are sorted based on the earliest link exit time and afterwards leave the link accordingly. Thus, this approach allows faster vehicles (lower $\left.t_{\text {earliest }}\right)$ to overtake (pass) slower vehicles (higher $t_{\text {earliest }}$ ) in uncongested regime and in the congested regime i.e. after queue formation, the vehicles follow the FIFO model [25]. Hereafter, this model is referred as "passing queue model".

$t_{\text {earliest }}=\frac{\ell_{l}}{\min \left(v_{l, \max }, v_{v, \max }\right)}$ 
In this contribution the passing queue model is further modified in order to allow passing of faster vehicles by slower vehicles in capacity and congested regime, which is referred to as seepage behavior as explained in "Introduction". Thus, this work proposes the "seepage queue model", which allow passing of slower vehicle by faster vehicles in the uncongested regime and vice versa in congested regime. The general approach for seepage functionality is similar to the approach in the previous study by Agarwal and Lämmel [9]. But, in the present study, the seepage queue model is improved and extended as shown in Algorithms 1 and 2. links are not violated. Seepage is not allowed between two vehicles of same type.

\section{Fundamental Diagrams}

In this section, the seepage queue model is validated with the help of fundamental diagrams (FDs). Car, motorbike and bike travel modes are used for the mixed traffic experiments in the present study. For simplicity, the passenger car units (PCU) and speeds of these modes are taken

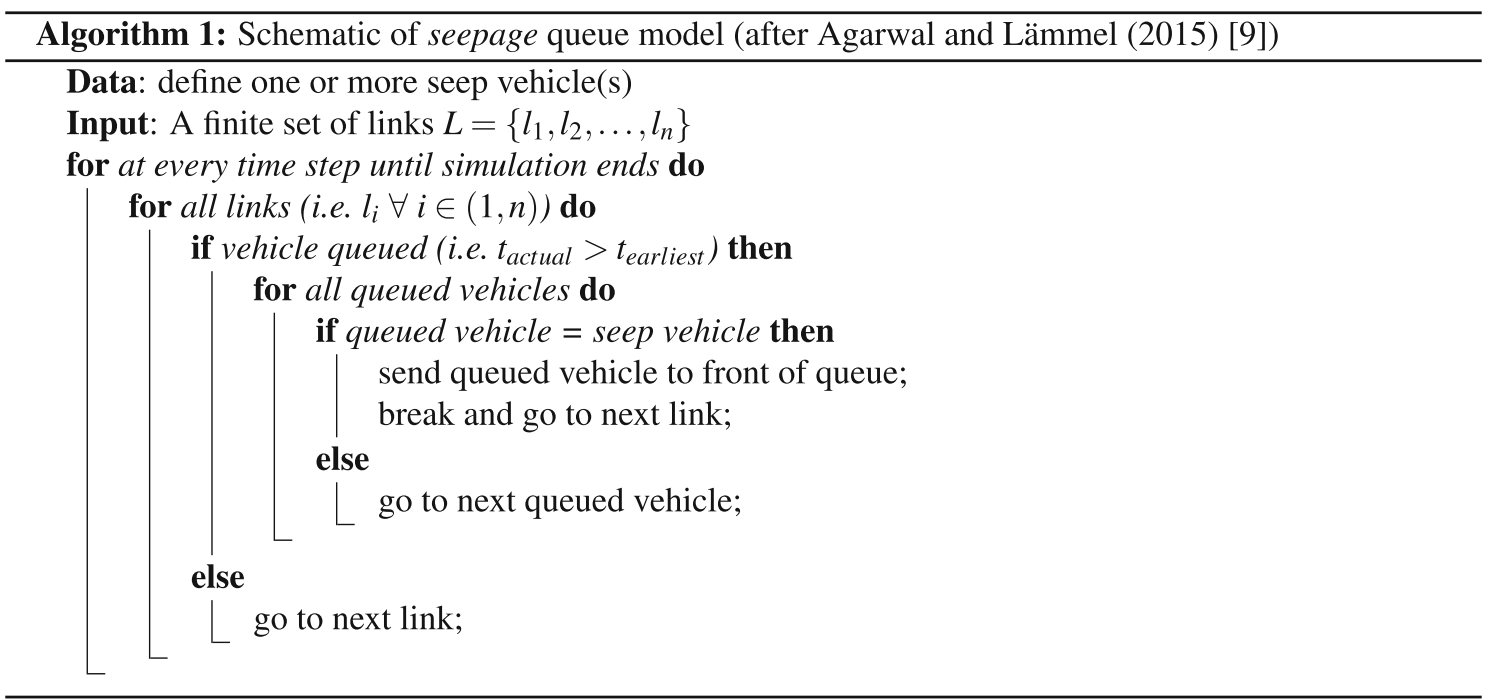

1. Instead of defining only one seep mode, the present study introduces the possibility to set multiple seep modes. Since, in the free flow regime, faster vehicles can overtake slower vehicle and therefore, passing is also allowed on the link.

2. If the flow on a link exceeds its flow capacity, a queue appears. Thus, in the simulation framework, the agent is queued if the time step $(t)$ exceeds the precomputed earliest link exit time ( $\left.t_{\text {earliest }}\right)$ of the agent on the link.

3. In the next step, just before a vehicle is about to leave the link, the vehicles whose earliest link exit time has passed (basically queued vehicle), are identified.

4. For identified vehicles, a seep mode is searched and if it is found then it is pushed to the front of queue and, afterwards, the front vehicle (seep mode) leaves the link depending on the flow capacity of the current link and the storage capacity of the next link.

5. If a seep mode is not found in the queue, flow dynamic remains unaltered i.e. first vehicle in the queue leaves the link if flow and storage capacities of the involved from the previous studies [25, 28] and are shown in Table 1 .

\section{Experiment Set Up for FDs}

In order to plot FDs, a triangular ${ }^{2}$ race track network is used as shown in Fig. 2. The race track network is the same as in previous studies [9, 25, 28]. The length of the each link in the network is $1000 \mathrm{~m}$. Maximum flow capacity and density for each link are $2700 \mathrm{PCU} / \mathrm{h}$ and $133.33 \mathrm{PCU} / \mathrm{km}$ respectively. All agents depart from left and continue rotating on the track until flow becomes steady and afterwards agents arrive on right.

\footnotetext{
The idea behind this experimental set up is to run fixed number of agents on the track until certain termination criteria is reached. A triangular race track is the simplest network to achieve and demonstrate this functionality, however, any other network may also be chosen.
} 
Table 1 Travel mode parameters

\begin{tabular}{lll}
\hline Travel Mode & PCU & Speed $(\mathrm{m} / \mathrm{s})$ \\
\hline Car & 1 & 16.67 \\
Motorbike & 0.25 & 16.67 \\
Bike & 0.25 & 4.17 \\
\hline
\end{tabular}

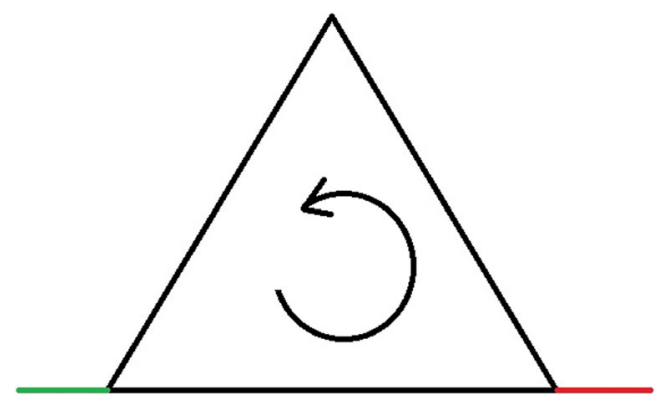

Fig. 2 Triangular race track network

\section{FDs with Backward Traveling Holes}

The FDs for the FIFO queue model and passing queue model that are presented in the previous studies [25, 28], do not display the typical triangular shape, since the congested branch of the FD is not well reproduced. For that reason the concept of backwards traveling holes [26] is used into the seepage queue model. The backwards traveling holes approach mimics the simplified Kinematic Wave Model (KWM) [29-31]. However, a true consistency with the theory is unclear since mixed-traffic and seepage is beyond the "classical" KWM. The backwards traveling holes approach works basically as follows (c.f. Agarwal et al. [26] for further details).

Every time a vehicle leaves the downstream end of a link the space freed by the leaving vehicle forms a gap or 'hole'. This 'hole' is not available instantly on upstream end of the link instead it is subsequently occupied by the following vehicles until it reaches the upstream end of the link. Consequently, it takes some time for the hole (space) to reach to the upstream end of the link $[32,33]$. Therefore, after a certain density has passed, no vehicle can enter the link until free space reaches the upstream end of the link and thus, the holes effectively introduce an inflow link capacity, in addition to the already existing outflow link capacity. Holes are distinguished by vehicle types. Therefore, two attributes are assigned to each hole: (1) size of the hole, which is same as the PCU of the leaving vehicle and
(2) speed of the hole, which is a constant for all vehicle types. Thus, each hole has precomputed times of arrival on the upstream end of the link. In the present study, the backward traveling hole speed is assumed to $15 \mathrm{~km} / \mathrm{h}$ which corresponds to a time headway of about $1.8 \mathrm{~s}$ between two subsequent vehicles.

\section{FDs for Only One Seep Mode}

For the reference purpose, the initial unclear seepage FDs from the previous study [9] are also presented here. Figure $3 a, b$ show the different FDs from passing and seepage queue models for equal model split (in $P C U$ ) of car and bike.

Figure $3 \mathrm{a}, \mathrm{b}$ show the FDs for passing and seepage queue models respectively. In Fig. 3a, the left branch of the FDs determined by the free flow (maximum) speed of the vehicle and maximum allowed speed on the link (see Eq. 1) and the right branch is determined by the fixed speed of the holes. The branches of FDs in free flow regime in Fig. 3a, b are same since, until the capacity regime is reached, the slope of the flow density curve is given by the minimum of maximum allowed link speed and maximum speed of vehicle (see Eq. 1). After that, flow starts decreasing until the flow becomes zero thus capacity regime together with the jammed regime governs the link dynamics during congestion. The speed-density plot shows the variation in the speed of the vehicle over total density. In the free flow regime, speed of car mode and speed of bike mode are equal to their maximum speeds and decrease afterwards.

From Fig. 3b, it can be observed that bike flow increases until a density of about $110 \mathrm{PCU} / \mathrm{km}$ and bike speed reduces marginally after free flow regime. On the contrary, car flow and speed is approaching zero at a lower density compared the passing queue model. But, data points corresponding to overall density higher than $110 \mathrm{PCU} / \mathrm{km}$ are not observed due to the flow dynamics for higher density. Data points in the race track experiment are only collected if flow and speed fluctuations are dampened. The fluctuations in the speed and flow are inspected when a vehicle leaves the base link (see Fig. 2). At higher densities, more bikes are in the queue and due to seepage behavior, only bike leaves the link. Since, car does not leave the link, car flow has higher fluctuations and subsequently, no data point is recorded for such state. This explains the missing data points after a density of $110 \mathrm{PCU} / \mathrm{km}$. 

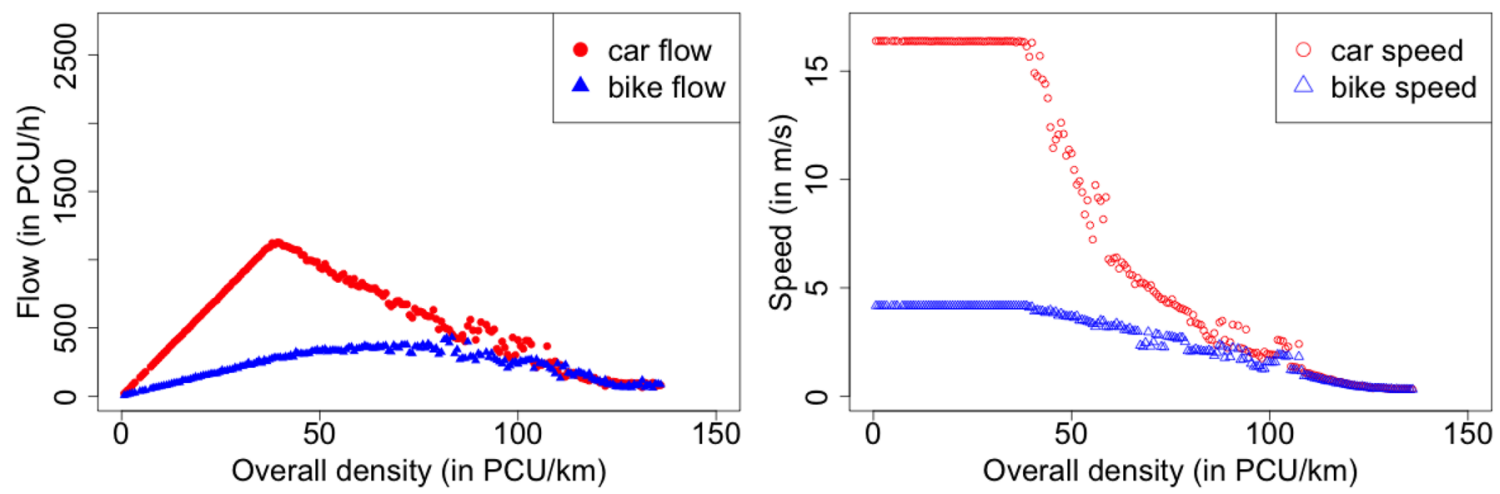

(a) Passing [26, 9]
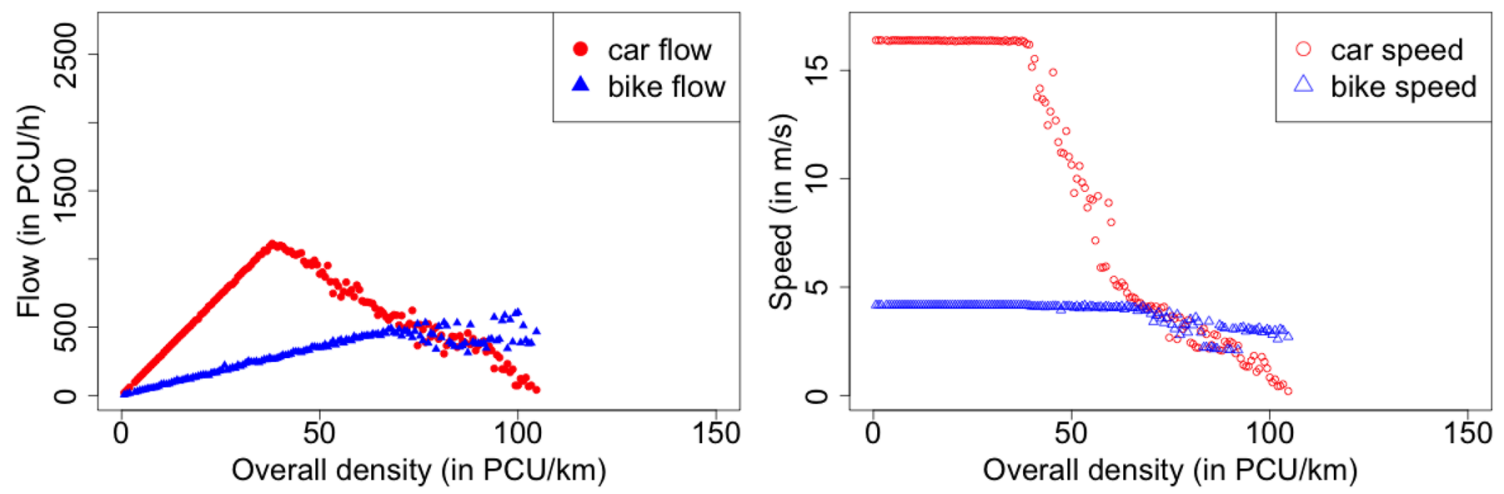

(b) Seepage [9].

Fig. 3 Fundamental diagrams for passing and seepage for car, bike simulations

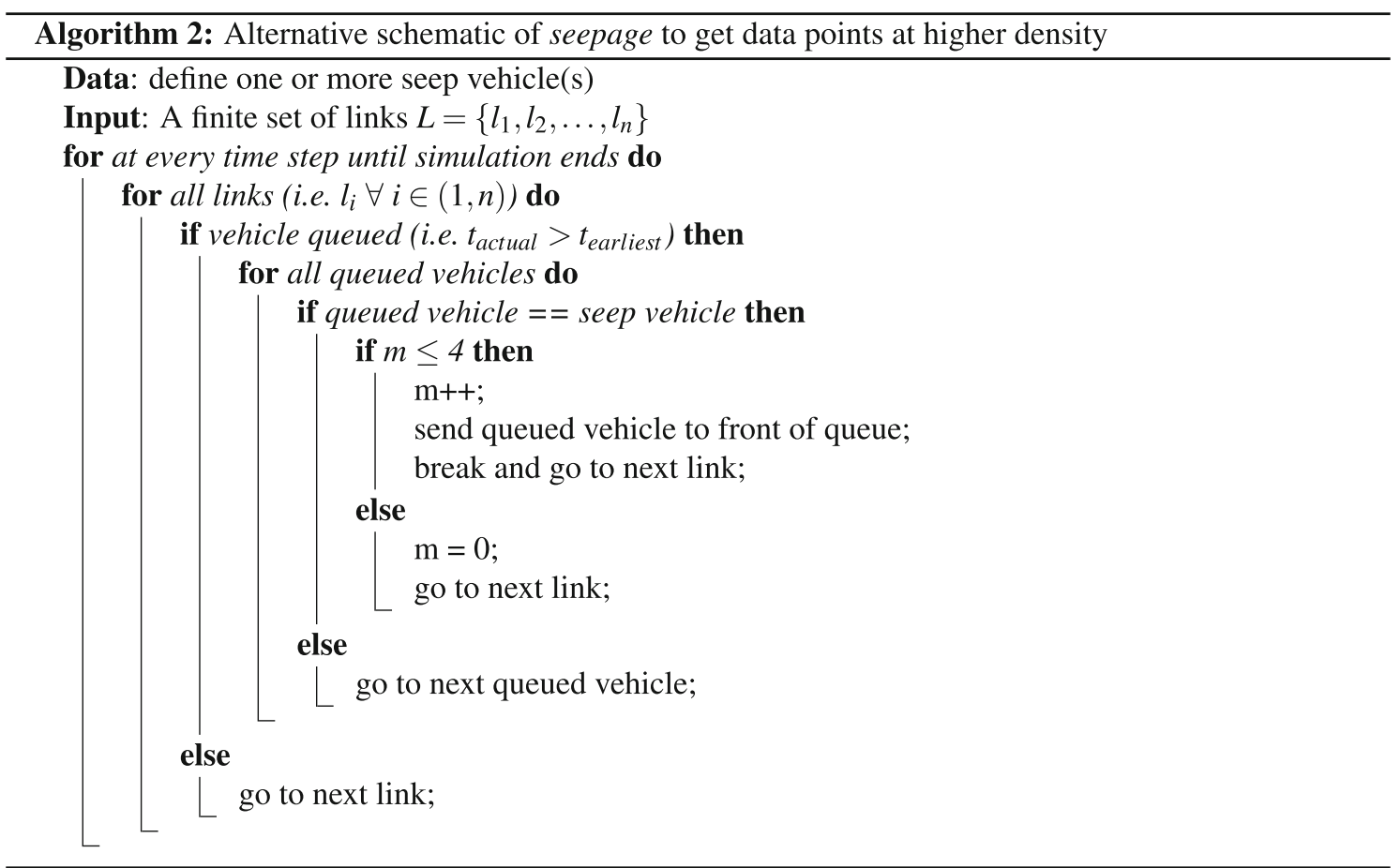



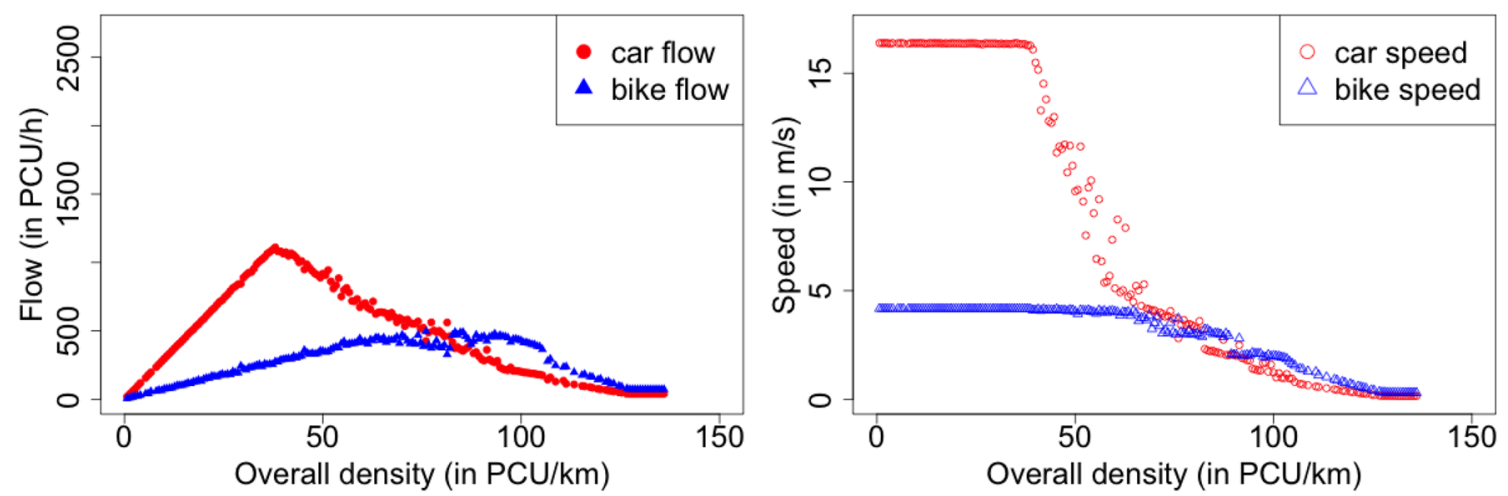

Fig. 4 FDs for seepage queue model according to Algorithm 2. Car and bike modes are taken in equal modal split and bike is considered as seep mode

\section{Restricted Seepage}

To get a more clearer congested branch of the FDs, the seepage behavior is restricted as shown in Algorithm 2. Since, even in the practical situations, all smaller vehicles do not perform seepage and therefore, the following process is reasonable. After every four ${ }^{3}$ bikes, the first vehicle (car or bike) in the queue is allowed to leave. The resulting FDs are shown in Fig. 4. Now, comparing Figs. $3 \mathrm{~b}$ and 4, it is more clear that, due to seepage, bike flow and speed start decreasing at a density higher than in the passing queue model. Thus, even after the seepage behavior is restricted, the speed of the bike in the seepage FD remains equal to the maximum speed of the bike up to a density of 65 PCU/ $\mathrm{km}$.

Clearly, in case of passing behavior (see Fig. 3a), flow characteristics of bikes and cars are affected by the presence of each other. On the contrary, in the seepage behavior (see Fig. 4), the flow characteristics of bikes are marginally affected by presence of cars but flow characteristics of cars are significantly affected by presence of bikes and thus producing a behavior similar to what is observed in reality. These results are in line with observations on traffic in developing nations where mixed traffic has smaller vehicles in abundance as shown in Fig. 1a.

\section{Multiple Seep Modes}

Since, due to its small size, motorbike also has high maneuverability and therefore can show the seepage behavior as shown in Fig. 1a. Therefore, in the present study, FDs are also plotted for mixed traffic situation where bike and motorbike both perform seepage behavior.

\footnotetext{
${ }^{3}$ In order to show the congested regime of flow-density curve of bike, this number is assumed to 4 (equivalent to $1 P C U$ ), however, the true value need to be calibrated from the scenario specific survey data.
}

Figure 5a, b show the FDs for passing and seepage queue model where car, motorbike and bike modes are simulated in equal modal split (in PCU). Motorbike and bike are defined as seep modes and Algorithm 2 is used to generate FDs. Again, similar to the Fig. 3a, b, the left branch of the FDs for car and motorbike are same because this branch represents free flow regime.

The speed of car and motorbike are same and therefore these two vehicles have overlapping data points in FDs for passing queue model (see Fig. 5a). Due to slower maximum speed of the bike mode, the left branch of the FD for bike has a flatter slope than the left branch of the FD for car and motorbike mode. The FDs for seepage queue model are shown in Fig. 5b. Clearly, after the density exceeds the free flow regime, the flow and average speed of car is smaller than flow and speed of motorbike respectively because motorbike can seep now. Similarly, bike mode also perform seepage but due to its lower maximum speed, only a marginal effect can be observed in the FD. Thus, if the modal share of faster seep mode is significant, the slower seep mode is not able to seep significantly.

\section{Sensitivity}

The most of the FDs shown in Fig. 3 are plotted for equal modal split and therefore, it is important to see the sensitivity of the seepage behavior. Hence, a sensitivity test is conducted to test the seepage behavior for different bike shares using Algorithm 2. Experiments are performed on the same race track (see Fig. 2). Two modes, car and bike are used for the simulation runs with different bike shares in the traffic mix. The speeds of car and bike modes are plotted against densities of car and bike respectively (see Fig. 6).

From the Fig. 6, one can observe that an increase in the bike share (a) decreases the density at which the speed of car starts decreasing and (b) increases in the density at 

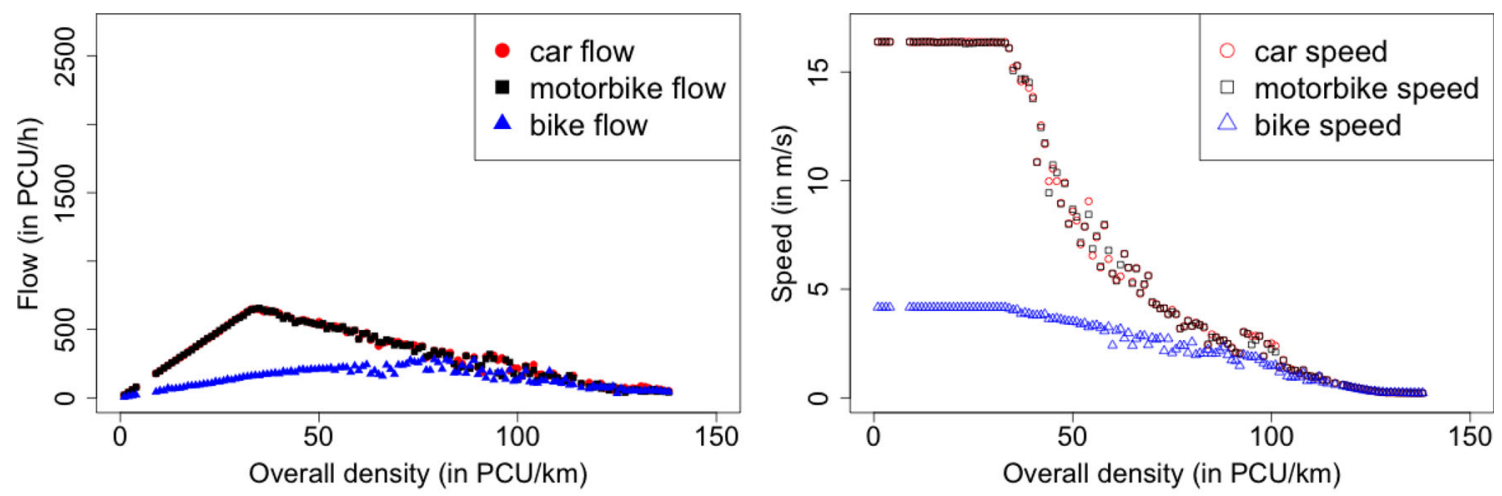

(a) Passing.
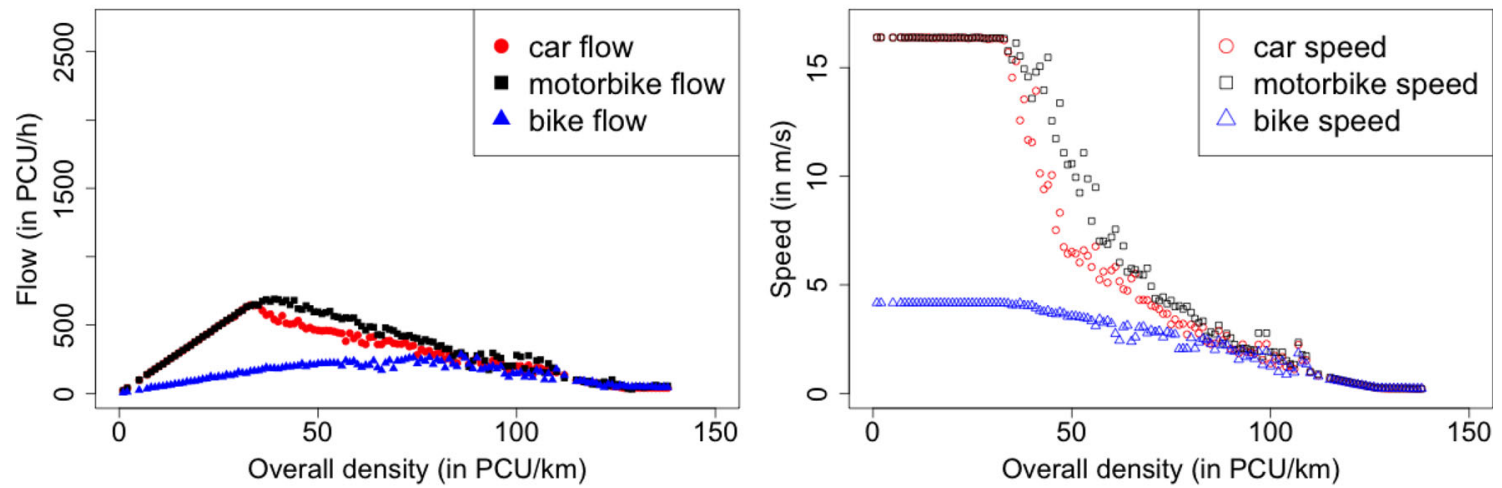

(b) Seepage.

Fig. 5 Fundamental diagrams from passing and seepage queue model for equal modal split (in PCU) of car, motorbike and bike simulations. Bike and motorbike are taken as seep modes
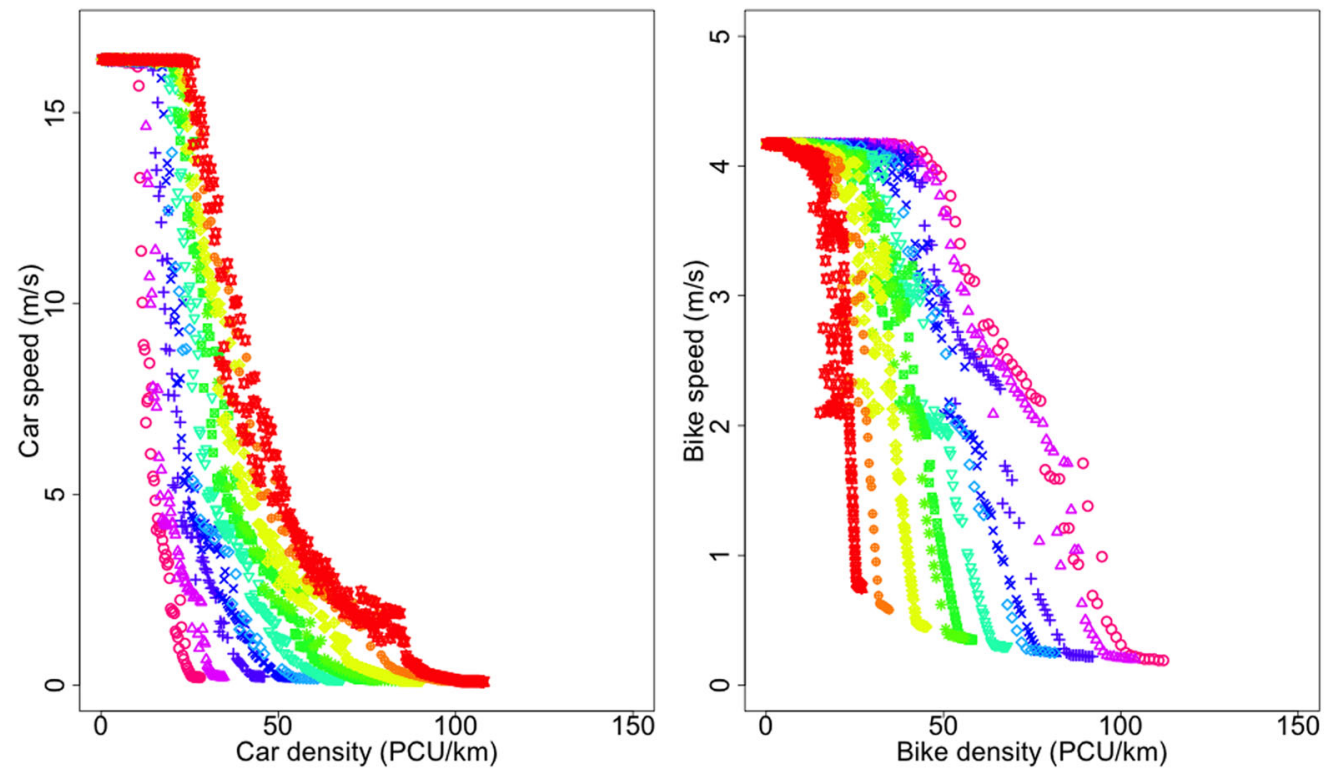

Modal split

in PCU

(car:bike)

- $1: 4$

$\triangle 1: 3$

$+\quad 1: 2$

$\rightarrow \quad 2: 3$

$\diamond 3: 4$

$-\nabla \quad 1: 1$

$-4: 3$

* $3: 2$

$\longrightarrow 2: 1$

- $3: 1$

4:1

Fig. 6 Variation in car and bike speeds for different modal splits in PCU 
which the speed of bike starts decreasing. This happens because, at a higher bike share, bike appear in front of the queue more often and consequently frequency of seepage of bike is higher. Hence, change in the speed of bike is marginal until higher density. Therefore, the resulting plot is plausible and similar to the behavior observed in reality.

\section{Application}

This sections describes the application of seepage in a large-scale transport simulation. The proposed seepage queue model is applied to a real-word scenario of Patna, India. The scenario is about an evacuation of heterogeneous traffic where seepage behavior is present.

\section{Real World Evacuation}

As described previously, in the "Introduction", a small experiment is conducted within the project Last-MileEvacuation [14]. The experiment study the evacuation of pedestrians using seepage action while moving through a group of stationary cars [15]. This is a static example but with the help of the proposed model, the evacuation of mixed traffic with seepage behavior is possible and presented next.

The following discusses the application of the proposed model a real-world evacuation scenario of mixed traffic with seepage behavior. The objectives of presenting this real-world scenario are to show and to quantify the influence of seepage for disaster management. The approach is useful for simulating large-scale evacuations (e.g. in case of tsunamis or forest fires) in densely populated areas where seepage is expected to take place.

Inputs The initial scenario is the same as in the study of Agarwal et al. [28] and therefore described here briefly. The input network consist of 3505 nodes and 7542 links. A disaster prone area is identified as evacuation area. The aim is to evacuate all the persons inside this area. The network is connected with some exit links which lead to a safer location.

The locations and travel modes of the persons are taken from the Patna comprehensive mobility plan [34]. For simplicity, it is assumed that all persons start evacuating simultaneously as soon as warning is announced and all persons start evacuating from their home locations. Thus in the simulation run, all persons inside the evacuation area are considered for evacuation. Assuming everyone starts at once is a conservative assumption, since it would lead to a high initial load onto the network and thus to high densities resulting in a lower throughput (cf. FDs in Fig. 3a, b) compared to widely distributed departure times. A study that investigates the influence of departure time distribution on the overall evacuation performance is presented in [35]. In absence of travel schedule for public transport (PT), PT mode is not considered in the simulation. The walk mode cannot be simulated using regular vehicular traffic model and therefore skipped in the simulation. Thus, similar to the previous study [28], car, motorbike and bike modes are considered in the simulation. Overall about $1 \%$ sample size is taken. Though, both motorbike and bike can be assumed as seep modes but for the simpler analysis purpose, only bike is considered as seep mode in this simulation run.

Simulation set up Two simulation runs are considered, one corresponds to passing and the other to seepage behavior. Simulations are run for 100 iterations. Until the 75 iterations, $10 \%$ of agents are allowed to change their route and remaining agents until 75 iterations and all agents after 75 iterations, select a plan from their generated choice sets according to probability distribution which converges to multinomial logit model [36]. In the 0th iteration, basically the shortest path is assigned to each agent between its origin and destination. Afterwards, agents learn and adapt to the system as described in the "Travel demand simulator: MATSim". Finally, the outcome of the last iteration shows the routes corresponding to an approximately Nash equilibrium (NE). Therefore, based on the agents' behavior, results are also analyzed for two cases, namely (1) shortest path (SP) (2) nash equilibrium (NE).

Results Table 2 shows the comparison of average trip time from two simulation runs and for both cases. Clearly, as expected, the average SP trip time is significantly higher than for $\mathrm{NE}$ in both simulation runs. Interestingly, the effect of seepage behavior can be observed in the 0th iteration only. For the SP case, the average trip time for bike with seepage behavior is about $13 \%$ less than the average trip time with passing behavior only. Consequently, due to seepage of bike, the average trip time of car and motorbike is increased.

In the NE case, the average trip time for each mode is significantly shorter than the average trip time in SP case for both simulation runs. Furthermore, in the seepage run, bike can overtake car and motorbike in the congested regime thus bike is faster and therefore average trip time of bike for seepage is $17.6 \%$ less than the average trip time for passing run. As a consequence of seepage of bike mode, average trip time of car and motorbike is 38.5 and $40.5 \%$ higher than the average trip time for passing run respectively. Interestingly, in the end, the total clearing time to evacuate all agents stays the same when seepage is enabled.

Evacuation progress Figure 7 shows the evacuation progress for both simulation runs and for both cases. Since, only bike is allowed to seep, car and motorbike modes are combined together and referred as fast mode whereas bike 
Table 2 Comparison of passing and seepage evacuation runs for Patna

\begin{tabular}{lllllll}
\hline Travel mode & Number of evacuee & \multicolumn{2}{l}{ Avg trip time passing (min) } & & \multicolumn{2}{l}{ Avg trip time seepage (min) } \\
& & SP & NE & & SP & NE \\
\hline Car & 168 & 301.94 & 123.42 & & 368.42 & 170.91 \\
Motorbike & 1266 & 329.57 & 152.46 & & 451.76 & 214.33 \\
Bike & 3921 & 382.73 & 178.47 & & 333.47 & 147.00 \\
\hline
\end{tabular}

Fig. 7 Comparison of evacuation progress. Fast refer to car and motorbike whereas slow refer to bike

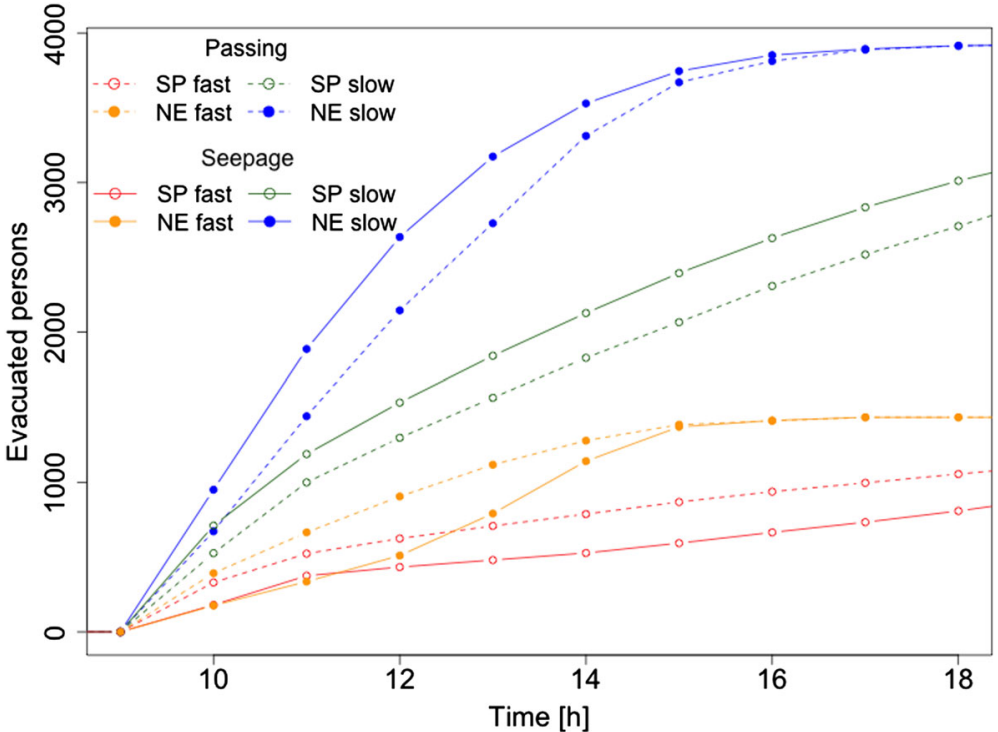

is referred as slow mode. Firstly, as expected, NE leads to shorter evacuation times compared to SP for both simulation runs and both mode types (slow and fast). The slow mode in the seepage run also shows the same trend. This is in line with the literature [16]. In terms of policy making this would mean the shortest path solution does not take congestion into consideration and thus it is not a feasible solution. But, on the contrary, due to seepage of bike, fast modes are stuck and therefore, in the fast mode of seepage run, initially, number of evacuated persons for NE (solid orange circles) is slightly lower than SP (hollow red circles). Furthermore, seepage has led to higher evacuation rate for bike mode and to lower rate for fast modes (car and motorbike). In the afternoon hours, fast modes in the seepage run catch up and become almost the same as for the passing run. Overall, seepage has no negative impact in terms of evacuation rate.

\section{Discussion}

In the present study, seepage of smaller vehicles bike (bicycle) and motorbike is studied. But, the same approach is applicable for any type of vehicle type across the world. Sometimes, car seep between the truck on a multi-lane highway. Seepage also plays an important role in situation where ambulance vehicles or other fire engines need to seep through large pedestrian crowds. This situation happens for example during large music festivals or other public events. In those situations the seep mode is not assigned to the smaller vehicle, instead the smaller "vehicles" (i.e. pedestrians) give space to the large vehicles (i.e. ambulances). Albeit those situations seem to be quite different from the seepage observed on road networks, it seems to be reasonable to apply a similar approach as the one that has been proposed in this contribution. The general applicability of queuing models to pedestrian traffic has been discussed in earlier works [37].

As shown in the Fig. 1b, during seepage in practical situations, bike (seep mode) do not occupy additional space instead use the space between the two cars in the same lane which in turn can relief the additional storage space. This additional space can enhance the saturation flow and overall result in lesser clearing time for evacuation scenarios. This functionality is not considered in the present study, but authors would like to implement it in the future studies.

In the present study, the parameter " $m$ " in Algorithm 2 is assumed arbitrarily. This parameter is scenario specific and therefore needs to be calibrated from the survey. The calibration of this parameter is beyond the scope of the present study. The experiments in the present study also need validation from the real-world survey data. 


\section{Conclusion and Outlook}

In order to simulate the heterogeneous traffic close to reality, this paper presented a so called "seepage" behavior in an agent-based simulation framework. This is a common praxis in most of the developing and some of the developed nations. Due to the smaller size and easier maneuverability, smaller vehicles (bike, motorbike) creep across the gaps between the stationary or almost stationary vehicles. The fundamental diagrams for various simulation runs were demonstrated for the validation of the proposed model. The dynamics of the queue model in the congested regime was unclear and therefore the concept of backward traveling holes was used which introduced a link inflow capacity implicitly. It was demonstrated that seepage is more effective for faster seep mode (for e.g. motorbike) rather than slower seep mode (for e.g. bike). The models sensitivity was tested by varying the share of bikes in a mixed traffic situation. A higher share of bikes led to a higher frequency of seepage actions and thus, seep mode retained its maximum speed even at higher densities.

Furthermore, the proposed seepage queue model was applied to a real-world scenario of Patna, India for the evacuation modeling in mixed traffic conditions. The passing and seepage queue model were compared based on this scenario. Clearly, the seepage behavior resulted in a significant decrease in average trip time for bike mode and in an increase in average trip time for car and motorbike modes. Overall, the total travel time for the seepage and passing simulation run were more or less same.

In future, the proposed model will be tested with traffic under regular conditions and for the system optimum solution for evacuation modeling, similar to Lämmel and Flötteröd [38], but for heterogeneous traffic conditions. Research into the application of the KWM theory to mixed mode traffic with seepage will be a future topic as well.

Acknowledgments The support given by DAAD (German Academic Exchange Service) to Amit Agarwal for his Ph.D. studies at Technische Universität Berlin is greatly acknowledged. The authors also would like to thank Kai Nagel at Technische Universität Berlin for his helpful comments and two anonymous reviewers for their suggestions. The authors acknowledge the opportunity to present the research work that forms the basis of this article at the 3rd Conference of the Transportation Research Group of India held at Kolkata (India) from 17-20 December, 2015.

\section{References}

1. Oketch T (2000) New modeling approach for mixed-traffic streams with nonmotorized vehicles. Transp Res Record: J Transp Res Board 1705(00-0285):61-69

2. Oketch T (2003) Modeled performance characteristics of heterogeneous traffic streams containing non-motorized vehicles.
Annual Meeting Preprint 03-0721. Transportation Research Board, Washington, D.C

3. FEMA (2009) A European agenda for motorcycle safety: the motorcyclists point of view. Tech. rep., Federation of European Motorcyclists Associations

4. Sperley M, Pietz AJ (2010) Motorcycle lane-sharing: literature review. Tech. Rep. OR-RD-10-20, Oregon Department of Transportation Research Section

5. Wang J, Yang J, Li Q, Wang Z (2004) The effect of nonstandard vehicle's special behavior on mixed-traffic modeling. Applications of Advanced Technologies in Transportation Engineering, pp 589-594

6. Asaithambi G, Kumar RVY, Sivanandan R (2013) Evaluation of exclusive stopping space for motorcycles at signalized intersections under mixed traffic conditions using simulation model. In: 92nd transportation research board annual meeting

7. Aupetit S, Espié S, Bouaziz S (2014) Naturalistic study of riders' behaviour in lane-splitting situations. Cognition, Technology and Work, pp 1-13

8. Centre for Road Safety (2014) Transport for New South Wales. Accessed 2014

9. Agarwal A, Lämmel G (2015) Seepage of smaller vehicles under heterogeneous traffic conditions. Procedia Comput Sci 52:890-895

10. Nair R, Mahmassani HS, Miller-Hooks E (2011) A porous flow approach to modeling heterogeneous traffic in disordered systems. Transp Res B: Methodol 45(9):1331-1345

11. Fan S, Work D (2015) A heterogeneous multiclass traffic flow model with creeping. SIAM J Appl Math 75(2):813-835

12. Fan S, Work D (2015) A cell transmission model for heterogeneous multiclass traffic flow with creeping. In: 94th Transportation Research Board Annual Meeting

13. MATSim webpage (2016) MultiAgent transport simulation. See http://matsim.org/. Accessed 2016

14. Taubenböck H, Goseberg N, Lämmel G, Setiadi N, Schlurmann T, Nagel K, Siegert F, Birkmann J, Traub K-P, Dech S, Keuck V, Lehmann F, Strunz G, Klüpfel H (2013) "Risk reduction at the "Last-Mile": an attempt to turn science into action by the example of Padang Indonesia", Nat Hazards 65(1):915-1945

15. Klüpfel H, Hebben S (2010) Numerical tsunami early warning system "Last-mile-Evacuation" WP 4100 : evacuation analysis and traffic optimization, evacuation plans for buildings and geometrically complex areas. Tech. rep., TraffGo HT GmbH

16. Lämmel G, Grether D, Nagel K (2010) The representation and implementation of time-dependent inundation in large-scale microscopic evacuation simulations. Transp Res C Emerg Technol 18(1):84-98

17. Dobler C (2013) Travel behaviour modelling for scenarios with exceptional events - methods and implementations. PhD thesis, ETH Zürich

18. Balmer M, Rieser M, Meister K, Charypar D, Lefebvre N, Nagel K, Axhausen K (2009) MATSim-T: architecture and simulation times. In: Bazzan A, Klügl F (eds) Multi-agent systems for traffic and transportation. IGI Global, pp 57-78

19. Balmer M, Raney B, Nagel K (2005) Adjustment of activity timing and duration in an agent-based traffic flow simulation. In: Timmermans H (ed) Progress in activity-based analysis. Elsevier, Oxford, pp 91-114

20. Raney B, Nagel K (2004) Iterative route planning for large-scale modular transportation simulations. Future Gener Comput Syst 20(7):1101-1118

21. Raney B, Nagel K (2006) An improved framework for large-scale multi-agent simulations of travel behaviour. In: Rietveld $\mathrm{P}$, Jourquin B, Westin K (eds) Towards better performing European Transportation Systems. Routledge, London, pp 305-347 
22. Gawron C (1998) An iterative algorithm to determine the dynamic user equilibrium in a traffic simulation model. Int $\mathbf{J}$ Modern Phys C 9(3):393-407

23. Simon P, Esser J, Nagel K (1999) Simple queueing model applied to the city of Portland. Int J Modern Phys 10(5):941-960

24. Cetin N, Burri A, Nagel K (2003) A large-scale agent-based traffic microsimulation based on queue model. In: Swiss Transport Research Conference (STRC), (Monte Verita, Switzerland). See http://www.strc.ch

25. Agarwal A, Zilske M, Rao K, Nagel K (2015) An elegant and computationally efficient approach for heterogeneous traffic modelling using agent based simulation. Procedia Comput Sci 52:962-967

26. Agarwal A, Lämmel G, Nagel K (2015) Modelling of backward travelling holes in mixed traffic conditions. In: Traffic and Granular Flow '15, (Delft, NL). Also VSP WP 15-15, see http:// www.vsp.tu-berlin.de/publications

27. Charypar D, Nagel K (2005) Generating complete all-day activity plans with genetic algorithms. Transportation 32(4):369-397

28. Agarwal A, Zilske M, Rao K, Nagel K (2013) Person-based dynamic traffic assignment for mixed traffic conditions. In: Conference on Agent-Based Modeling in Transportation Planning and Operations. Blacksburg, Virginia. Also VSP WP 12-11, see http://www.vsp.tu-berlin.de/publications

29. Lighthill MJ, Whitham JB (1955) On kinematic waves. I: flow movement in long rivers. II: A theory of traffic flow on long crowded roads. Proc. Roy. Soc. A 229:281-345

30. Richards P (1956) Shock waves on the highway. Oper Res $4(1): 42-51$

31. Newell G (1993) A simplified theory of kinematic waves in highway traffic. I: General theory. II: Queueing at freeway bottlenecks. III: Multi-destination flows. Transp Res B 27B:281-313
32. Charypar D, Axhausen K, Nagel K (2007) Event-driven queuebased traffic flow microsimulation. Transp Res Record 2003:35-40

33. Eissfeldt N, Krajzewicz D, Nagel K, Wagner P (2006) Simulating traffic flow with queues. See http://www.vsp.tu-berlin.de/ publications

34. TRIPP, iTrans, and VKS (2009) Comprehensive mobility plan for Patna urban agglomeration area. Tech. rep., Department of Urban Development. Government of Bihar

35. Lämmel G, Klüpfel H (2012)Slower is faster: the influence of departure time distribution on the overall evacuation performance. In: International conference on evacuation modeling and management. Northwestern University, Evanston. see also vsp$\mathrm{wp}=11-29$

36. Nagel K, Flötteröd G (2012) Agent-based traffic assignment: going from trips to behavioural travelers. In: Pendyala R, Bhat C (eds) Travel behaviour research in an evolving world-selected papers from the 12th international conference on travel behaviour research, ch. 12. International Association for Travel Behaviour Research, pp 261-294

37. Lämmel G, Klüpfel H, Nagel K (2009) The MATSim network flow model for traffic simulation adapted to large-scale emergency egress and an application to the evacuation of the Indonesian city of Padang in case of a tsunami warning. In: Timmermans H (ed) Pedestrian behavior, ch. 11. Emerald Group Publishing Limited, London, pp 245-265

38. Lämmel G, Flötteröd G (2009) Towards system optimum: finding optimal routing strategies in time-tependent networks for largescale evacuation problems, vol 5803 of LNCS (LNAI). Springer, Berlin, pp 532-539 